\title{
Responsibilities, role and integration of Mission Operations within the complex ground-segment of the German TerraSAR-X radar mission
}

\author{
P. Mühlbauer, H. Hofmann, M.P.Geyer, R. Kahle \\ German Space Operations Center (DLR-GSOC), D-82234 Wessling, Germany.
}

\begin{abstract}
TerraSAR-X is a high-resolution radar-satellite of a new generation to be launched in 2006. It will be Germanys first earth observation space project based on a public-private partnership with financial contribution from industry. Beside scientific utilization the project will open a large potential for the commercial exploitation of remote sensing data. These facts and the highly integrated ground-segment with significant contributions from three DLR institutes constitute the unique characteristic of this novel project.

The Mission Operations System (MOS) itself serves and operates the direct interface to the satellite not only during the mission lifetime but already in its AIT-phase by means of a compact but comprehensive Monitoring and Control System, invoking a number of new core-elements, tools, processes and concepts not only in the technical but also operational and management fields - see ref. [1]. Furthermore one of the major tasks within the MOS is the development, validation and operation of the Mission Planning System (MPS) which is designed to screen and evaluate, to plan, optimize and finally to realize a magnitude of data-take requests by making optimum use of resources while satisfying the scientific and commercial user community - all this on a time-scale aiming to set new benchmarks. Up- and downlinkencryption and on-ground key management, stringent manoeuvre requirements to keep the spacecraft on its nominal reference-orbit during the entire lifetime and the necessity to employ a new Monitoring- and Control system (MCS) for this demanding mission complete the project specific challenges.

In parallel to these demands posed by the mission and system requirements a large number of technical and operational interfaces with the Instrument Operations and Calibration Segment (IOCS - responsible for radar instrument operation and calibration) and with the Payload Ground Segment (PGS - responsible for payload data reception, processing, archiving and distribution) leaves the MOS in complex interaction within the ground-segment, which itself interfaces with the commercial marketing company and its customers. On the background of all this, the paper will address benefits and synergies but also challenges and sensitive areas from the MOS point of view, attempting special analysis on and trying to convey lessons-learned and provide an outlook for follow-on missions.
\end{abstract}

Rome, Italy - June 19-23, 2006A 1 of 19 


\section{Introduction}

Peter Mühlbauer - M.S. in Aerospace Engineering from the Technical University of Munich - works with the German Space Operations Center since 1984 in the area of mission operations of geo-synchronous and low-earth satellites as well as human space-flight missions onboard the former MIR space-station. Since 1999, the responsibility of project-management for mission operations preparation and -conduct for three overlapping earth observation satellite missions has resulted in valuable experiences especially in the field of multi-mission and multi-satellite operations.

Presently the authors main responsibility is as project-manager of the Mission Operations System for the TerraSAR-X mission supported by the co-authors as project system-engineers. They all are part of the integrated satellite multi-mission team at GSOC which also operates the German national mission CHAMP and the American/German cooperation GRACE - both highly successful. The team is dedicated to apply the lessons-learned from the past [ref. 1] and to incorporate the new concepts and processes described in this paper to make TerraSAR-X a milestone in secure and cost-effective mission-operations in a new type of future cooperative, complex and increasingly commercial space projects.

\section{$\underline{\text { TSX - Project, - Mission and Mission Operation System Overview }}$}

- German high-resolution X-Band Radar Mission (Flexbus-spacecraft by EADSAstrium)

wide range of scientific and commercial applications

Public-Private-Partnership (co-funding)

- Launch with Ukrainian launcher DNJEPR in October 2006

- Operation in sun-synchronous orbit on 515 $\mathrm{km}$ altitude for 5,5 years

- Strict orbit-control (+/- $250 \mathrm{~m})$ during entire mission on this "reference orbit"

- Tasks of DLR-GSOC:

o Development of Mission Operation System (MOS)

o Development and operation of "Central Checkout System" (CCS) for S/C-AIT support

o Satellite operations and mission planning during lifetime

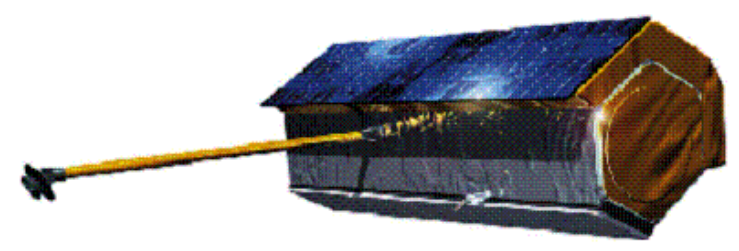

Fig. 1 - TerraSAR-X spacecraft

$\begin{array}{ll}\text { Orbit type } & \begin{array}{l}\text { Sun-synchronous } \\ \text { repeat orbit } \\ 11 \text { days } \\ \text { Repeat period }\end{array} \\ \begin{array}{l}\text { Repeat cycle } \\ \text { Orbits per day }\end{array} & \begin{array}{l}15+2 / 11 \\ 18: 00 \pm 0.25 \mathrm{~h}\end{array} \\ \begin{array}{l}\text { Equatorial } \\ \text { crossing time }\end{array} & \begin{array}{l}\text { ascending pass } \\ 0.0011 \div 0.0012 \text { frozen }\end{array} \\ \text { Eccentricity } & 97.443823 \text { deg } \\ \text { Inclination } & 90 \text { deg } \\ \text { Argument of } & \\ \text { perigee } & 514.8 \mathrm{~km} \\ \text { Altitude at the } & \\ \text { equator } & \\ \text { Semi-major axis } & 6892.9 \mathrm{~km}\end{array}$

Table 1: TS-X orbit characteristic 


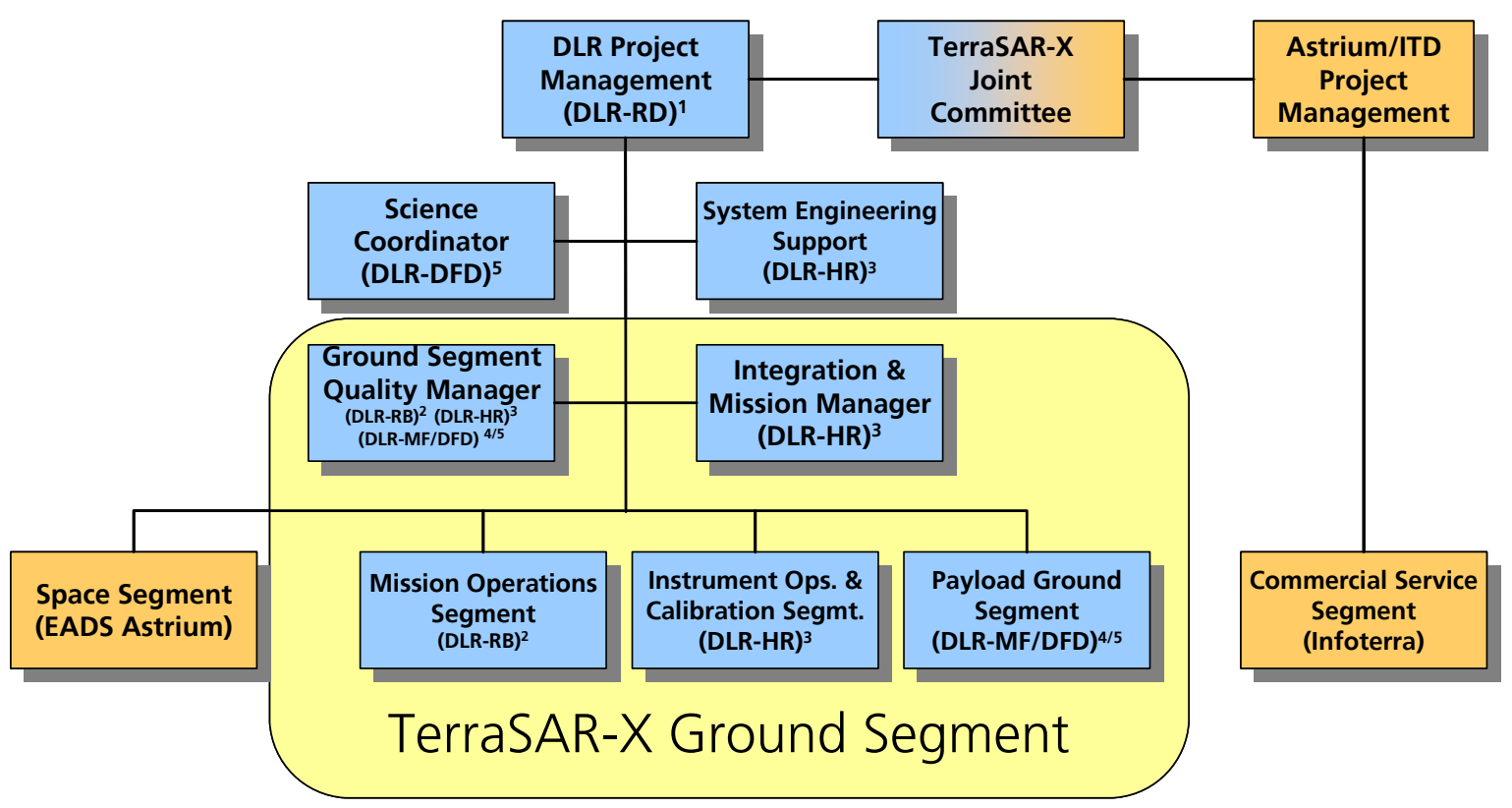

Fig. 2 - TSX cooperative management structure (DLR responsibility in blue)

The high-resolution radar satellite mission TerraSAR-X makes high demands on its ground segment. The German Space Operation Center (GSOC) in Oberpfaffenhofen, which is part of the German Aerospace Center (Deutsches Zentrum für Luft- und Raumfahrt e.V., DLR), will operate TerraSAR-X during the envisaged mission time of 5 years. The TerraSAR-X project is financed by a public private partnership between DLR and EADS (Astrium/Infoterra).

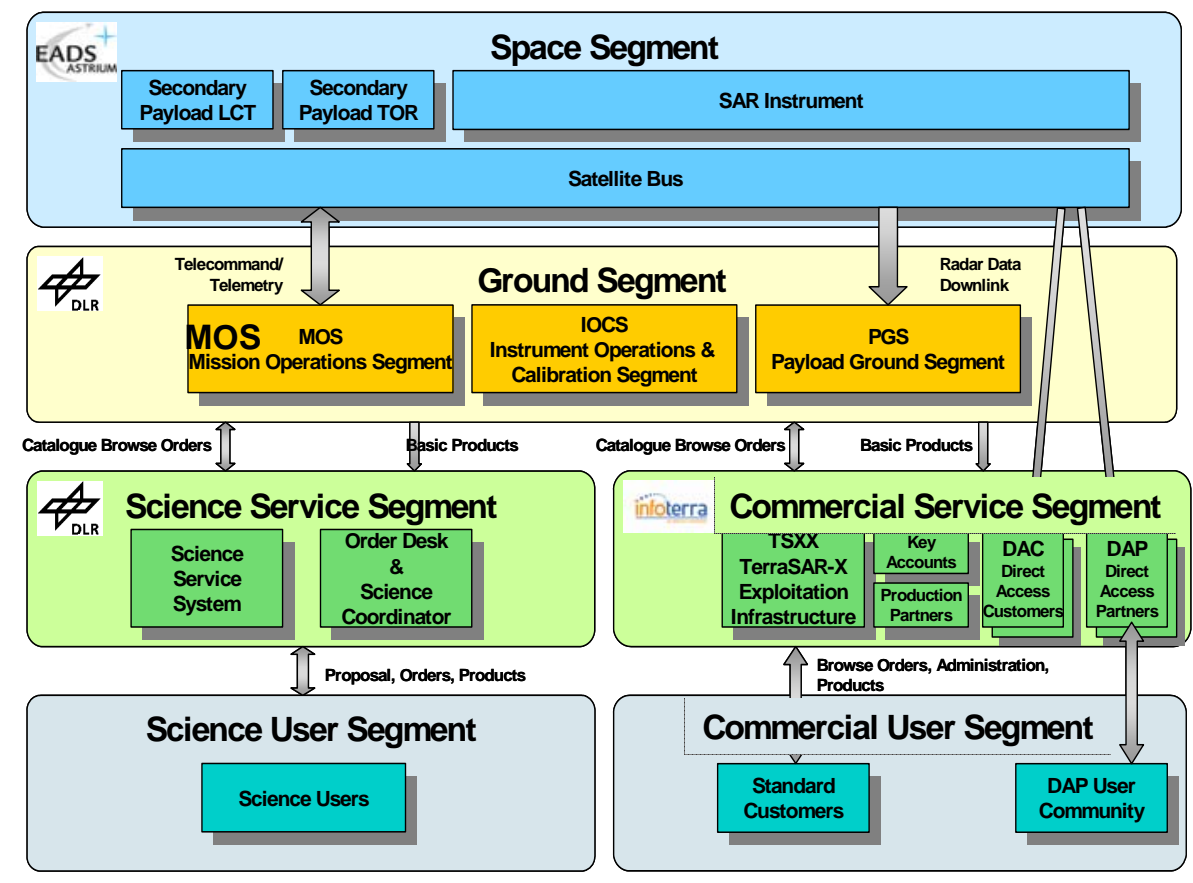

Fig. 3 - TerraSAR-X project functional organisation and overall interfaces 


\section{SpaceOps 2006 - Conference}

The main payload of the single satellite mission is an X-band synthetic aperture radar (SAR). Envisaged earth observation applications are geographic information products like topographic maps, infrastructure planning, crop stand measurements, flood damage assessment and geological structure maps. Other proposals include ship detection services, oil spill monitoring and traffic investigations. Secondary payloads are the high-precision GPS receiver TOR (Tracking, Occultation and Ranging) for precise orbit determination and the experimental Laser Communication Terminal LCT.

The SAR system has three prime modes: "ScanSAR" provides $100 \mathrm{~km}$ and "Stripmap" $30 \mathrm{~km}$ across track product coverage size with less than $3 \mathrm{~m}$ or $16 \mathrm{~m}$ geometric resolution, respectively. The geometrical order length is variable. "Spotlight" provides the smallest product coverage sizes, 5-10 x $10 \mathrm{~km}$ with a geometric resolution of less than $1 \mathrm{~m}$.

The Mission Operation Segment (MOS) is one part of the TerraSAR-X Ground-Segment (G/S) established by DLR, together with the Payload Ground Segment (PGS) and the Instrument Operations Segment (IOCS) - all of them provided by three institutes within DLR with different history and background with either scientific or more operational emphasis and "working-culture".

The MOS functions as the acting interface to the spacecraft, not only in orbit but also during the spacecraft AIT-phase on ground [1]. It consists of the standard elements like ground-station network, Monitoring and Control System (MCS), communication networks, flight dynamics and operations facilities.

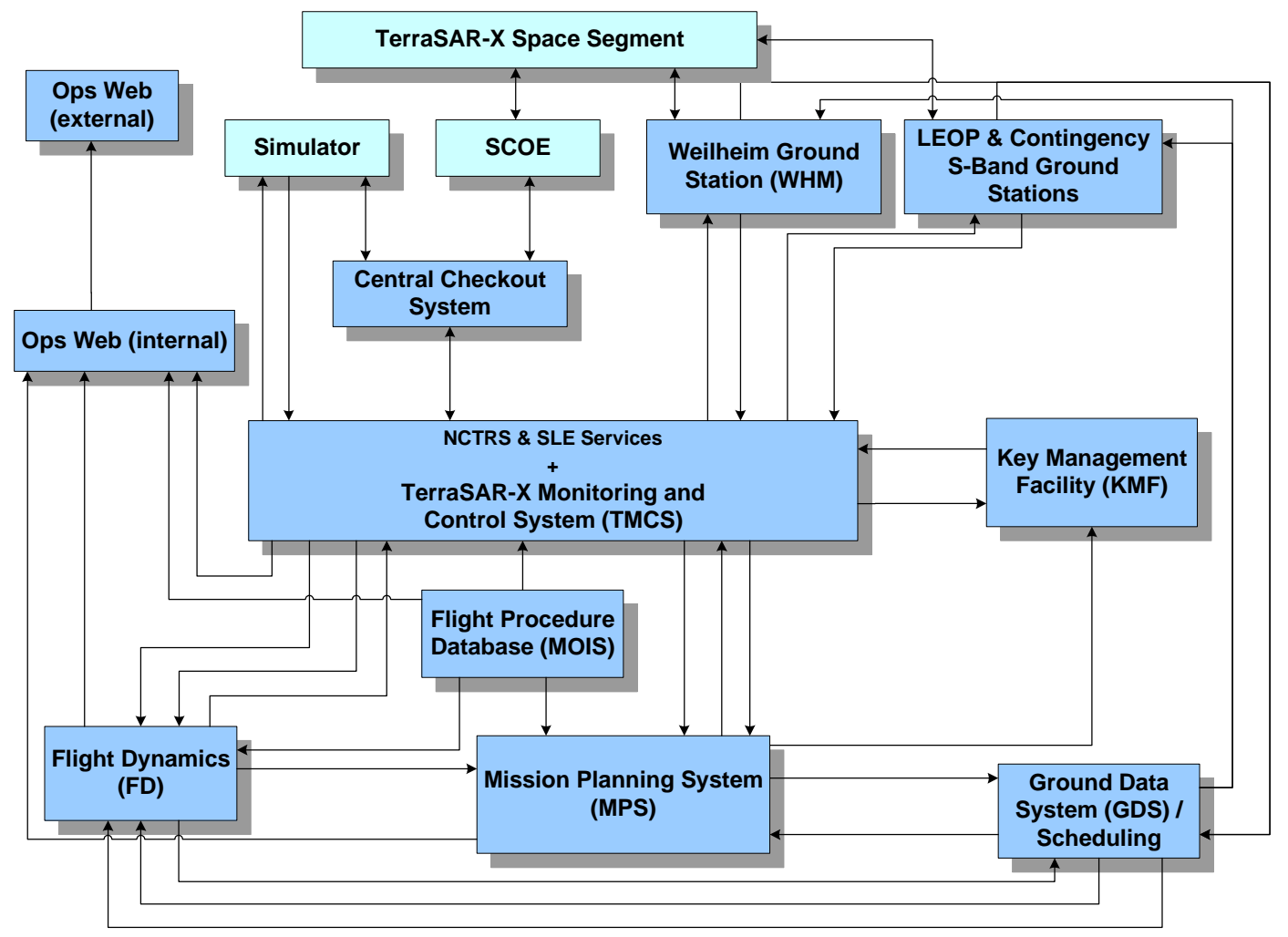

Fig. 4 - Mission Operation System (product- and interface-names omitted for confidentiality) 
Furthermore, the unique TSX mission requirements resulted in a number of project-specific features which in scope and interaction are new to the spacecraft operations environment at GSOC. These four main areas are briefly outlined below because their challenges mainly contribute to the large number of interfaces and the complex processes:

\section{1 - Mission Planning:}

The TerraSAR-X satellite resources are designed for carrying out about $\mathbf{5 0 0}$ datatakes per day. The user may choose between "single orders" with one defined set of geographical coordinates or "coverage orders", where a specified geographical region is split automatically into atomic single orders by mission planning. "Standing orders" allow the user to requests periodical datatakes in a given time frame from one geographical target.

The common mission planning task consists of collecting requests for satellite payload operations and matches them with the availabilities and resources of the system. The end product is a feasible and optimized plan that takes into account all constraints of both space and ground system. This plan must be distributed to the other parts of the system.

The expected amount of user requests for the TerraSAR-X payload system gives crucial requirements for the design of the ground system. The mission planning system will be dimensioned to cope with an overload of about $100 \%$, that is more than $\mathbf{1 0 0 0}$ datatake requests per day must be handled. Challenging time constraints from the user side demand that mission planning accepts low priority tasks as late as three days and high priority tasks as late as 6 hours before the daily planning cycle for the next planning horizon [ref. 5].

During the scheduling process, the system automatically tracks the on-board resources using numerical models. Actual telemetry data is used to update the calculated results. Among other things this includes the mass memory and command buffer used, electric power, link capacities from and to ground stations and a simple power/thermal model of TerraSAR-X.

\section{2 - Security}

A specific aspect of the TerraSAR-X mission is the necessity to ensure the confidentiality of the acquired radar data as well as securing the communication to the satellite. These tasks are - on ground - handled by the so-called Key Management Facility (KMF). The KMF authenticates and/or encrypts the telecommands which are sent to the S/C where they are received by the onboard decryption module. For the second task the KMF generates the specific encryption keys, which are used to encrypt the SAR data and sends these to the S/C. The respective decryption keys are provided to the auxiliary data pickup point, from where the receiving ground stations can retrieve only their key(s). For security reasons the decryption keys are itself encrypted. 


\section{3 - Monitoring and Control System (MCS) - including Central Checkout System (CCS):}

The following major tasks were faced for setting up an almost completely new Monitoringand Control system for the TerraSAR-X mission:

- Substitution of the old MCS as used for missions CHAMP and GRACE required due to new and more complex TSX-mission requirements.

- New core MCS supplied by SCOS-2000 (assigned goal by the program "network-ofcenters" in context of European cooperation together with CCSDS standardization issues). In the course of design and implementation, the ever increasing project requirements showed that this was a decision well made since the old system would not have been capable to handle them. The transition to new MCS for routinely supported missions (CHAMP, GRACE) is presently ongoing

- Comprehensive changes in operating systems and hardware-platforms (compatibility and maintenance during routine operations phase as major issues to be considered)

- Development and management of common Scos2000-based Telemetry- and Command Database (MIB) in cooperation with spacecraft manufacturer [ref. 1]

- New version of TLM-data display system (SATMON) to be incorporated and accepted, offline data processing system and a large variety of tools for data and performance analysis to be developed or significantly upgraded or adapted

- Integration of MOIS as tool for procedure development and management - also extensively used for spacecraft AIT

- "Central Checkout System" (GSE for spacecraft AIT) to be provided to and operated for the spacecraft manufacturer parallel to the MCS-development within GSOC [see ref. 1]

- Implementation of SLE/NCTRS services for interfacing between control-center and ground-stations

In all the main- and core-functionalities unfortunately no or very little heritage could be drawn from the previously prepared and still flown missions. This challenge developed into one of two major critical issues during the TSX-MOS project preparation (see below). 


\section{4 - Orbital Control and Flight Dynamics}

During the entire mission the spacecraft shall follow the same trajectory relative to the Earth (i.e. relative to an Earth fixed rotating coordinate system). One way to achieve this is to prescribe a certain target trajectory for the spacecraft to follow. This target path is the so-called reference orbit, see [2] for details on its generation. Once such a reference has been defined, the actual osculating orbit shall be kept within certain bounds representing the limits of the deadband for the orbit control.

Those bounds are derived from the SAR interferometry, which is based on the stereoscopic effect that is obtained by matching two SAR images obtained from two slightly different orbits. This off-set creates an "interferometric baseline", i.e. up to $500 \mathrm{~m}$ for TerraSAR-X. For dedicated interferometric operations (e.g. differential interferometry) the baseline is reduced up to $60 \mathrm{~m}$ for selected orbit arcs.

Keeping the spacecraft within such a tube of $\mathbf{2 5 0} \mathrm{m}$ or even $\mathbf{3 0} \mathrm{m}$ radius (with its center line being the reference orbit) demands a high accuracy orbit control from a ground-in-the-loop point of view. To achieve this, the actual satellite trajectory has to be determined precisely. This is done by using on-board GPS raw measurements for Precise Orbit Determination, in order to get the initial condition for prediction of the satellite trajectory. The difference between the predicted and the reference orbits is the baseline for the planning of necessary orbit control maneuvers to keep the satellite within the $\pm 250 \mathrm{~m}$ (or $\pm 30 \mathrm{~m}$ ) tube with a minimal number of maneuvers.

Additionally, the precisely determined trajectory (i.e. $20 \mathrm{~cm}$ 3D position accuracy) is delivered to the user community in order to support exact processing of the SAR data.

The mission requirements pose a big challenge for the ground-in-the-loop orbit control of TerraSAR-X. Due to expected high frequency of maneuvers (up to 1 per day during Solar Maximum), it is planned to automate the whole process. An adequate Flight Dynamics System which fulfills all mission requirements has been analyzed in different studies, e.g. see [3] and [4], and its implementation is nearly completed. For details on the TSX orbit characteristics see table 1. 


\section{MOS-integration into and interaction within the ground-segment}

In Fig. 5 the overall interface management concept within the project is outlined. "Interface Control Documents- ICD" define all the technical, operational and sometimes even management processes and interfaces in relation between the ground-segment and the external partner (satellite, users, ..) but also within the ground-segment among the three involved sub-segments.

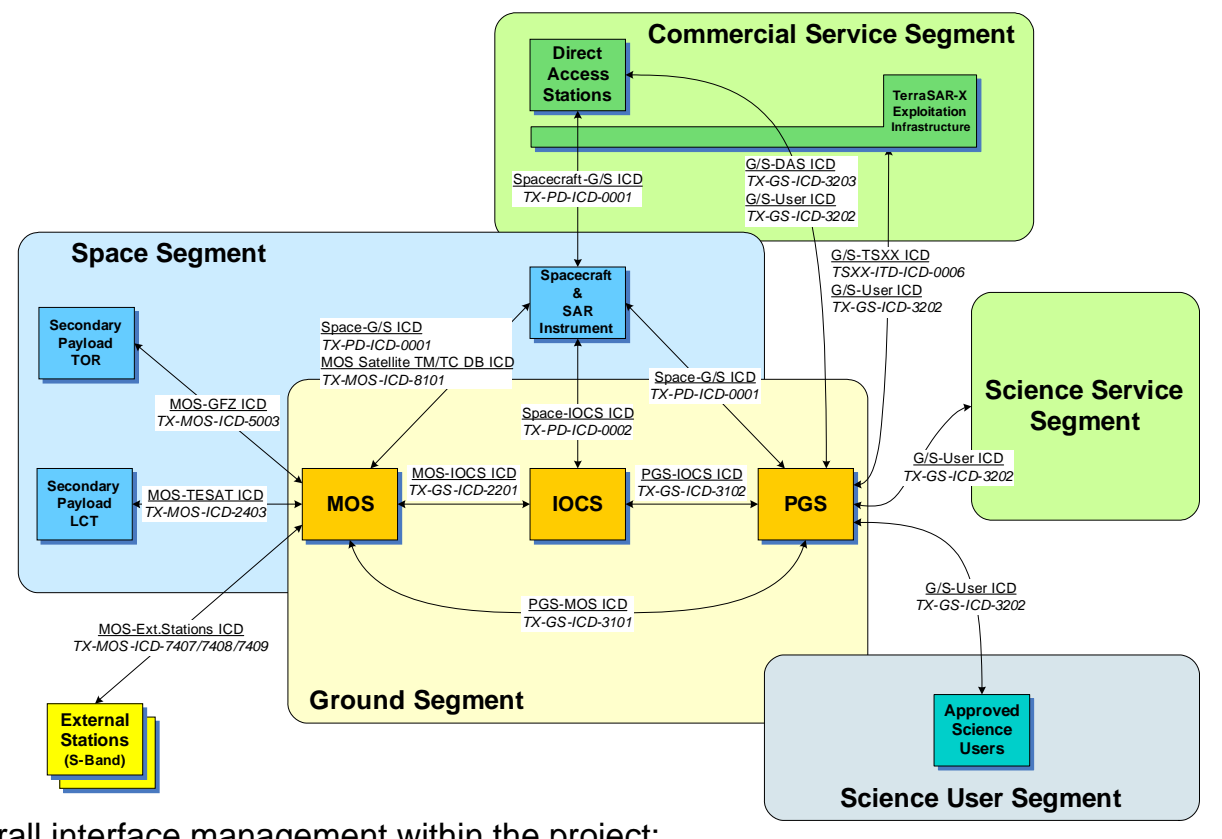

Fig. 5 - Overall interface management within the project:

In the course of ground-segment integration and the planning for verification and validation of such a complex network with this variety of interfaces and products, a central database system was established by the PGS (Payload Ground Segment) for the G/S. Under the supervision and authority of the "Integration- and Mission Manager" (IMM) - see fig. 2 - all ground-segment relevant processes and databases are established and maintained. It supports all QM on ground-segment level (documentation management as well as configuration control) but also integration and test planning, execution support and reporting. Its extension with an operational anomaly- and recommendation handling tool for the operations of the ground-segment after launch is presently in progress.

Not only from the MOS point of view this concept has proven to be very valuable and effective in supporting all interface-, integration- and test relevant processes and issues. Therefore also all the MOSfacilities, -elements and -products with external interface or significance are managed and controlled (CM) on this level and with this system. The use of one common system for G/S-integration- and test as well as quality-management and configuration-control by all otherwise quite independent subsegments is highly recommended to ensure efficient communication, interaction and compatibility. 


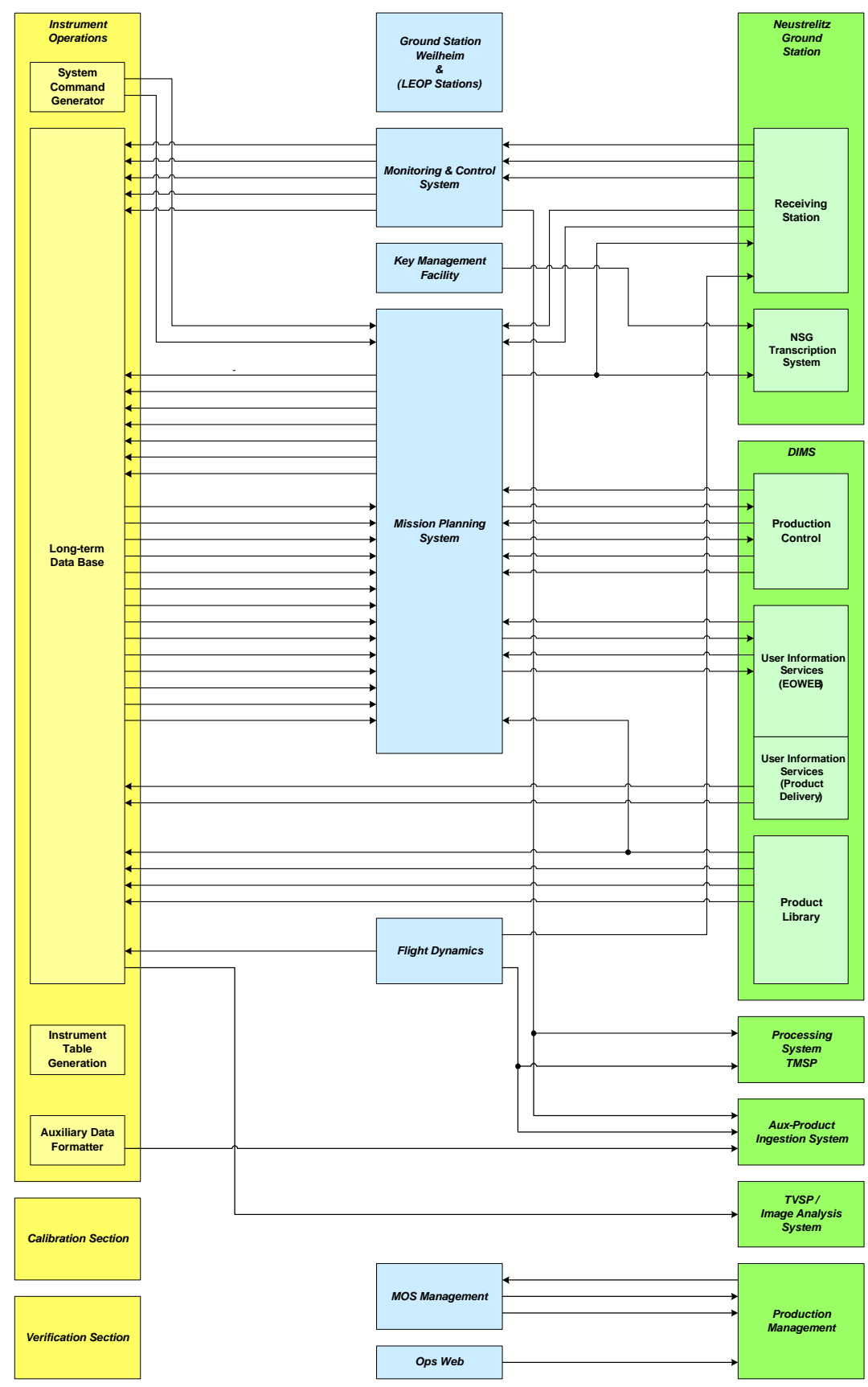

Fig. 6 - MOS-integration (center) within ground-segment components and its interfaces

This figure not only lists the main-elements of these sub-segments but also quite clearly displays all the technical and operational interfaces between them. Product- and interface-names are not published here for confidentiality reasons.

As it can be seen, the MOS is the central element of the TSX-ground-segment with respect to the number of interfaces and incoming and outgoing products. In addition, the central position of the Mission Planning System within the entire ground-segment is demonstrated.

Rome, Italy - June 19-23, 2006A 9 of 19 
Specific tasks and responsibilities of the MOS within the ground-segment

The following specific tasks mark the areas where the responsibility for coordination and management lies with the MOS:

- Interface to the spacecraft and the radar instrument in orbit and during AIT on ground

- MOS is central point for operational validation of ground-segment and satellite because of the application of the "Integrated Concept" [1] in cooperation with S/C manufacturer (Training \& Simulation Plan for operational validation of G/S plus spacecraft provided and executed by MOS). The driver is the satellite AIT but overall coordination is done by MOS.

- MOS plays main-role in definition and implementation of operational processes, facilities and environments to ensure continuous operation and maintain $S / C$ health and safety

- Identification and resolution of conflicts between orders and/or maintenance and maneuvers is done by during the planning process by MOS

- Maintain and operate the spacecraft within its tight operational boundaries (orbit, attitude, maneuvers) to ensure payload operation and data-validity

- Provide and manage operational and data security for the entire project

Due to the central position of the MOS (fig. 6) and its critical tasks it is - on the other hand - quite vulnerable to schedule conflicts, delivery delays of critical items or externally imposed drastic requirements changes. As a general rule can be stated: The more interfaces and the more critical tasks may be allocated to a subsystem, the more sensitive it is to disturbances of any kind and the more critical this is for the rest. This was observed clearly in the phases of implementation and integration of the Mission Planning System (MPS).

\section{Interfaces, products and processes}

The complexity of the TerraSAR-X ground segment, which on the one hand results from the fact, that it is composed of three segments - provided from three different institutes - and on the other hand from the specific requirements concerning the payload operations, demanded already from the beginning of the project particular efforts for interface definition and implementation.

This started with the need to develop a common "language" between the three parts (IOCS, PGS and MOS), which had all their own history of how to accomplish a project. Consequently, with respect to the interfaces the first task that was performed, was the definition of an "interface methodology" document describing exactly how the interfaces have to be specified. This ensured 
a common way of defining the interfaces not only between the three segments, but was also used for the segment-internal interfaces and to the external users.

Having solved the "language" problem between the segments the next step was the actual definition of the (new) interfaces connected with the payload operations. A major part in this respect plays the mission planning system (MPS). Being the one entity that plans the datatakes, it has interfaces with the order and processing elements at PGS as well as with the IOCS, which has to provide the correct radar parameters for the datatakes. Since the order process of a datatake is done in real-time - within the next minute(s) the user will get feedback about costs and feasibility - the order processing and evaluation has to be done in R/T as well. Consequently real-time connections between MPS and the counterparts had to be established and several technical, operational and administrative problems to be solved.

The R/T interface with IOCS for the radar parameter calculation was realized by including this specific part of software directly into the mission planning. To do this, ICOS specified a prototype, which was then re-coded into the MPS software, while the configuration tables are still provided by the IOCS long-term data base. Experience during the last years showed, that this approach is connected with several problems and a high amount of coordination. A better approach would probably have been to completely program the IOCS S/W part at IOCS and then integrate the whole S/W en block. This is in discussion for the follow-up mission(s).

The non real-time interfaces between MPS and its partners mainly provide information about the planning process (which runs twice a day and includes the newly received orders) and the detailed data needed for the ground stations and processing centers about the datatakes once they are finally planned (e.g. reception time of a certain datatake at a certain ground station).

Since the ground segment is connected to the science and commercial costumers, a specific interface issue is the registration of users and the later provision of the necessary data. Acting as external interface for the whole ground segment is PGS, but also MOS has to register new users to provide additional information like orbit and attitude data, decryption keys or housekeeping telemetry. Therefore a registration form and process has been created, which serves both PGS and MOS needs and allows them to integrate new users into their systems. For data access two pickup points were established: one at PGS allows access to the products, the other at MOS allows access to all auxiliary data as described above (keys, etc.).

In general the integration of the MOS into the TSX ground segment with the described boundary conditions was a process that took much more time and effort for coordination than estimated. The following numbers taken from figure 6 may serve as illustration of the complexity: 
- Overall number of MOS interfaces within G/S only:

$50-60$

- Overall number of products exchanged within G/S:

$50-60$

Data and operational security requirements, encryption and key-management

As written earlier there are specific security requirements with respect to S/C commanding and data confidentiality, which for the MOS are handled by the key management facility. S/C commanding is an internal MOS process, which is transparent for the ground segment. For data confidentiality however a common concept in the form of two pickup points were established that provide the processed SAR data on the one, and all auxiliary products on the other pickup point. To ensure the confidentiality both pickup points can only be reached by secure ftp transfers (sftp/ftps) and are located within the DLR wide area network.

\section{Challenges in the development of the mission planning system (MPS)}

The development and integration of the Mission Planning System (MPS) developed into the first critical area in the TSX-MOS project:

- Novel mission and planning concept (Public-Private-Partnership, commercial/scientific conflict of interests, priority regulations under constant discussion, short-term businesscase adaptations by commercial partner, absolutely challenging time-constants for planning-process and short-term reaction and implementation times...) [see ref. 5]

- Significant delivery delays: Instrument-command- and radar-parameter-generator prototypes (input for core-elements of MPS) were still under development while modules should have already been coded and accepted for implementation to support integration and test. Subsequent changes after delivery of versions 1 had to be considered.

- A host of important and design-driving requirements to the MPS still evolved during MOS phase $\mathbf{D}$ (implementation and even integration) for the following reasons:

o The design work on ground-segment level effectively resulted in a variety of new requirements to the MPS - and all this at a stage where the mission planning system was already under implementation on basis of the overall project requirements known so far!

o Discussion on planning-issues with the commercial service provider and project partner began at the same time where MPS-implementation was already ongoing.

- These negative effects were wide-reaching because MPS is the central element in the G/S with key functionalities and -tasks and a record number of interfaces and products 
has to be handled with internal and external partners => highly sensitive to interfacechanges, test-failures or delivery-delays

Fortunately effective counter-measures were taken on-time so that this highly critical issue could be solved.

\section{Acceptance and validation of new Monitoring and Control System - MCS}

The second critical issue had to be faced in the area of development and acceptance of the almost completely new Monitoring and Control System. The integration of the MOS into the G/S with the MCS being the another important subsystem of the MOS - was not an immediate cause for the encountered problems, but in the other direction the delay in integration and acceptance of the new TSX-MCS affected the G/S-integration and -testing process during project phase D2 and could only be minimized by workarounds in combination with adaptations of the G/S-integration as well as -verification and -validation schedule.

For this reason it is mentioned here to benefit from the lessons-learnt for future complexly integrated ground-systems. Fortunately for the entire TSX-project, parallel launch-date adaptations relieved the critical situation for the MOS.

What were the processes to be managed and problems to be solved in the area of the MCS? The following tries to give a summary:

- Total effort and time required for adapting the new but generic MCS (SCOS2000kernel) to the project requirements (including its acceptance and validation) and setting up specific tools, interfaces and databases and its overall complexity was severely underestimated. The entire process was extended by more than a year.

- $\quad=>$ Major challenge in the TSX-MOS project was thereafter the acceptance and validation of the new MCS as a process parallel to the operational use of the same MCS on the "Central Checkout System" (CCS) supplied by MOS to the spacecraft manufacturer for AIT-support!

- The immediate on-the-job verification and validation of the "virgin" MCS versions in immediate context of spacecraft AIT-support on the Central Checkout System (CCS) at the premises of the spacecraft manufacturer was not intended. This provided and in many cases provoked immediate feedback from the "battlefield". A proper and wellscheduled acceptance-process for such a central and important feature of a mission was not feasible. However constructive feedback was received and immediately integrated and without this challenge the whole process might have taken still longer.

Resulting recommendation: Critical or important systems must not be developed, accepted and validated on a platform or within a concept or a project which should already or in immediate 
future be available for or provide any kind of operational support! This also applies for subsystems to be incorporated into a larger ground-segment. The entire subsystem has to be fully accepted before releasing it for operations or entering into the next step of integrating the whole. 


\section{Recommendations for overall management between cooperation partners}

The following items mainly affect the cooperation between the MOS and the spacecraft manufacturer in case of close cooperation which is highly recommended to benefit from synergies. The experience from TSX-project however has shown that these issues are also encountered in context of the ground-segment integration between equal partners on a somewhat limited scale:

- Cooperation with S/C-manufacturer and/or other partners in the area of common tools, databases, procedures and testing-activities are highly recommended and provide rewarding spin-off and synergies [ref. 1]

- Define the understanding and implementation of "tailoring" of requirements (acc. to ECSS) on time and under mutual agreement with partners and review-board. This is especially true for the review of available documentation at certain milestones. In a cooperative approach where the MOS works together with the S/C-manufacturer or other partners certain deliverable documents may not be required at the formal delivery-dates according to standards. But this must not lead to a situation where this issue is neglected at all!

- Deliveries: responsibilities, schedule, content and scope need to be defined and documented sufficiently (FOPs, procedures, tools, parameter- and operational descriptions, simulator, prototypes, databases, parameter-limits, ...). It is the task of the overall project management to contractually impose such definitions and regulations.

- The same applies of course for all deliveries and contributions by the MOS (GSE ...)

\section{Recommendations for integration of a complex ground-segment among equal partners}

- Respect influence of different "working-cultures and languages": partners or institutes coming from different background sometimes show very different understanding of terms and working-culture. This may become very obvious between scientifically working institutes in the research field on the one side and operations and service-oriented facilities on the other side. Time and effort invested in clarifying these differences and making them aware to all partners are wisely spent.

- Overall and timely identification and implementation of responsibilities and workpackages required on ground-segment level (system- and performance engineering, quality- and configuration management, integration management, ...)

- System-engineering function is essential on G/S-level (responsible for performance issues; interface definition, -verification and -validation) 
- When integrating sub-segments into an overall ground-segment we are immediately faced with two non-congruent project levels (two or more sub-segments, one groundsegment) $=>$ these overlapping or sometimes subsequent project phases require exact distinction. In the TSX-G/S mission preparation the formal reviews for both the subsegments and the ground-segment were done in one process to make effective use of time and other resources - however this sometimes resulted in almost irresolvable discussions on review-goals, deliverable documentation and correctly applied "tailoring" of standards. The following recommendations derive from this experience (see fig. 7):

o Respect the sub-projects right for its own tailoring-approach and their different milestone schedule - there may be good reasons for it.

o Allow for non-parallel schedule on the level of the sub-segments if necessary.

o Allow for one review-step back-log of the G/S-schedule at least at the beginning of the project - the integration of the whole can never be as fast as the implementation of the elements !

o Pay extensive attention to where the different internal schedules may generate conflicts in other sub-segments (delayed deliveries from one to the other) and try to avoid such dependencies on critical input at all (specifications or deliveries)

o Accept the fact, that the final driver for completion of work in case of conflict will almost always be the sub-segment itself - and not the super-imposed groundsegment.

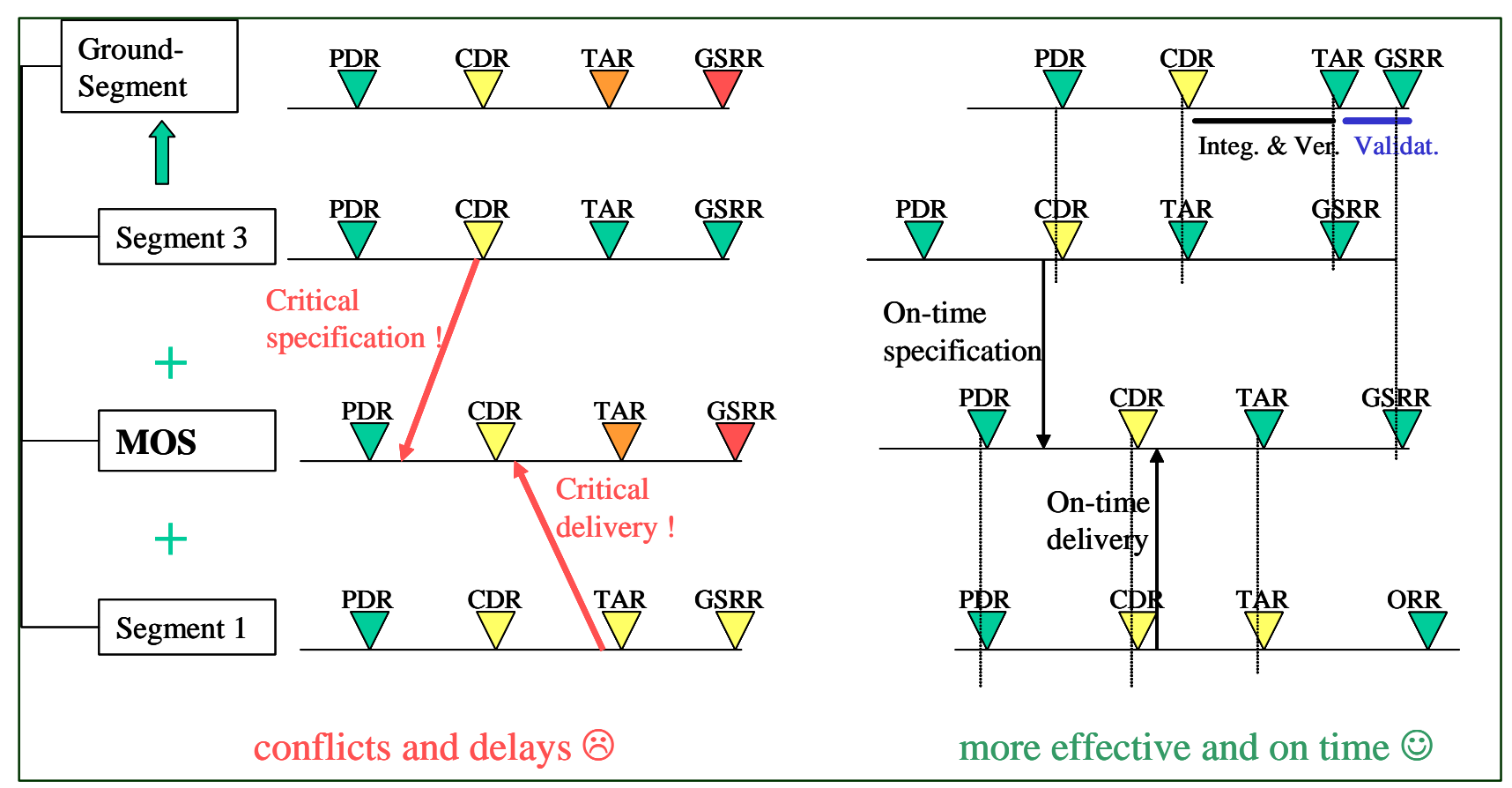

Fig. 7 - planning of project phases and review dates 
- Apply the correct (ECSS- or other standards) requirement to the character of the project and of the milestone to be reviewed! There are significant differences between softwareand operations-projects which are also considered by the ECSS (see ECSS-M-70). This is important in the field of required status for reviews and documentation deliveries.

- Definition of scope and schedule for G/S-internal deliveries including implementation of "contractual" agreements about handling of delays and significant changes which might cause disturbances on implementers side (recipient). This has to be managed and supervised by an authority (overall project-management) including on-time implementation of corrective action or counter-measures. Unless this is not taken care of, the negative consequences are left to the affected implementer with potential catastrophic consequences for the whole.

\section{Remaining Conclusions}

The MOS is the key interface to the spacecraft and the central element in terms of mission readiness, safety and operational proficiency. The following recommendations for the integration of complex groundsegments are given therefore purely from this point-of-view:

- Invest time and effort at the beginning of the project to understand and respect the different "working-cultures" and find a common language (in some areas this common understanding needs to be documented! - e.g. the "TSX - interface methodology document")

- Perform a bi-level, interleafed schedule planning from the beginning of the combined project. Observe the many specific schedule issues within each sub-segment when doing so and when defining the review-milestones requirements and its schedule !

- Observe the project-character (operational or software) when defining the "tailoring" of standards for the review-process, also for the schedules.

- Avoid critical deliveries at all or at least significant delays of such between the sub-segments

- Leave key work-packages and tasks in the segment with the relevant expertise, do not transfer them to other segments for schedule, resource or other reasons!

- In comparison to a "stand-alone project", provide at least $150 \%$ resources and time for interfacedefinition, -implementation, -verification and -validation within each sub-segment!

- In addition to this, provide system-engineering resources on the ground-segment level to take care of performance-issues, interface-definition, - integration, -verification and -validation.

- Use of common systems and tools for G/S-integration- and test as well as quality-management and configuration-control is highly recommended 
- The more interfaces and the more critical tasks may be allocated to a subsystem, the more sensitive it is to disturbances of any kind and the more critical this is for the rest !

- Expect the fact that during design and even early integration of the ground-segment several additional and important requirements for the sub-segments will be identified! This means that even during their phase D1 enough slack in schedule has to be provided to incorporate additional requirements without jeopardizing the entire schedule.

- Establish a project management plan that handles the potential risks of the ground-segment interfaces

- Last but not least the main message from the author with all the best wishes for TerraSAR-X and all of its potential follow-on projects:

Critical systems must not be developed, accepted and validated on a platform or within a concept or a project which should already or in immediate future be available for or provide any kind of operational support. This also applies for critical subsystems to be incorporated into a larger ground-segment. The entire subsystem has to be fully accepted before releasing it for operations or entering into the next step of integrating the whole system. Provide for sufficient time and resources to cope with any unforeseen challenge, in context with new developments and programs.

\section{References:}

[1] P. Mühlbauer, T. Kuch, W. Päffgen; Integrated Concepts for A Global Enterprise - ..., SpaceOps 2004 Conference Montreal (May 18, 2004)

[2] D’Amico S., Arbinger Ch., Kirschner M., Campagnola S.; Generation of an Optimum Target Trajectory for the TerraSAR-X Repeat Observation Satellite; 18th International Symposium on Space Flight Dynamics, 11-15 Oct. 2004, Munich, Germany (2004).

[3] Arbinger Ch., D'Amico S., Eineder M.; Precise Ground-In-the-Loop Orbit Control for Low Earth Observation Satellites; 18th International Symposium on Space Flight Dynamics, 11-15 Oct. 2004, Munich, Germany (2004).

[4] Arbinger Ch., D'Amico S.; Impact of Orbit Prediction Accuracy on Low Earth Remote Sensing Flight Dynamics Operations; 18th International Symposium on Space Flight Dynamics, 11-15 Oct. 2004, Munich, Germany (2004). 
[5] Michael P. Geyer, Armin Braun; Tailoring the TerraSAR-X Mission Planning System to PPP needs; SpaceOps 2006 Conference, Rome (June 19-23, 2006) 